\title{
Following autophagy step by step
}

\author{
Tom Egil Hansen and Terje Johansen* \\ See research article: http://www.biomedcentral.com/1741-7007/9/38
}

\begin{abstract}
Autophagy is an evolutionarily conserved lysosomal degradation route for soluble components of the cytosol and organelles. There is great interest in identifying compounds that modulate autophagy because they may have applications in the treatment of major diseases including cancer and neurodegenerative disease. Hundeshagen and colleagues describe this month in BMC Biology a screening assay based on flow cytometry that makes it possible to track distinct steps in the autophagic process and thereby identify novel modulators of autophagy.
\end{abstract}

Eukaryotic cells degrade proteins through two systems, the ubiquitin-proteasome system, and autophagy, whereby cytoplasmic components become enclosed in a double membrane to form a compartment known as the autophagosome and are delivered to the lysosome for degradation. Constitutive, basal autophagy occurs under nutrient rich conditions and serves as a quality control mechanism for both proteins and organelles, to protect the cell from the consequences of aggregated proteins and damaged organelles that could cause the development of disease: failure of this system is implicated in the development of, for example, neurodegenerative disease and cancer. Autophagy is also induced in response to starvation in order to generate nutrients and energy through the degradation of macromolecules and organelles [1,2].

\section{Autophagy and disease}

Research during the last decade has made it increasingly clear that autophagy plays important roles in most of the

*Correspondence: terje.johansen@uit.no

Molecular Cancer Research Group, Institute of Medical Biology, University of Tromsø, 9037 Tromsø, Norway

(C) 2011 Hansen and Johansen; licensee BioMed Central Ltd. This is an Open Access article distributed under the terms of the Creative Commons Attribution License (http://creativecommons.org/licenses/by/2.0), which permits unrestricted use, distribution, and reproduction in any medium, provided the original work is properly cited. major human diseases as well as in infection and immunity [1,2], with increasing evidence for selective autophagy of protein aggregates, organelles and pathogens [3]. Autophagy may, depending on the circumstances, either inhibit or promote tumor growth. Thus it may contribute to genomic stability by clearing cells of damaged organelles and protein aggregates that produce reactive oxygen species resulting in DNA damage; or on the other hand, it may help tumor cells survive stress conditions like hypoxia and nutrient deprivation [4]. Neurodegenerative disorders such as Alzheimer's, Huntington's and Parkinson's disease are characterized by the deposition of intra- and extracellular proteins aggregates that are not degraded by proteasomes. Thus, activation of autophagy is often observed in protein aggregation diseases and autophagy deficiency leads to neurodegeneration in mice and fruit flies. The life span of organisms as diverse as Caenorhabditis elegans and Drosophila and even mice can be significantly increased by boosting autophagy. Efficient autophagy may thus protect against neurodegeneration and increase longevity $[1,2]$.

Because of the involvement of autophagy in many disease conditions there is great interest in identifying drugs that can be used to manipulate autophagy for therapeutic purposes. An important step towards this goal is to establish assays and screening systems where the autophagic flux can be measured rapidly and precisely in response to drugs. Autophagic flux refers to the complete process of autophagy (Figure 1), which begins with the formation of a crescent-shaped double membrane, the phagophore, which expands around a portion of cytoplasm and fuses to form the autophagosome. The mature autophagosome then either fuses directly with a lysosome to generate an autolysosome, or fuses first with a late endosome to form an amphisome, which then fuses with the lysosome to give an autolysosome. In the autolysosome, the cargo is degraded. 


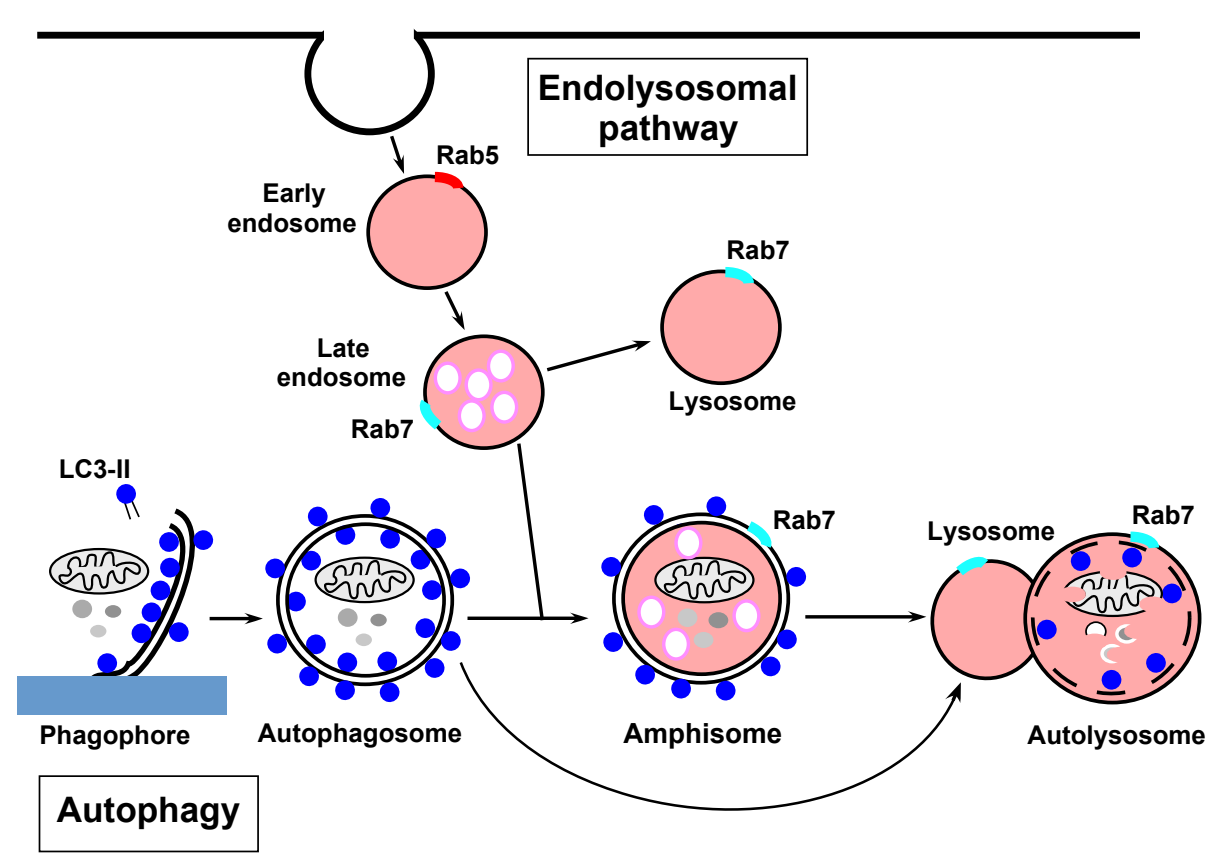

Figure 1. The autophagic and endolysosomal pathways intersect and interconnect. Schematic drawing showing the autophagy process with phagophore and autophagosome formation followed by fusion of autophagosomes either directly to lysosomes to form autophagosomes, or to late endosomes to give amphisomes that subsequently fuse with lysosomes. Lipidated LC3-II, used as a marker for autophagy, is tightly associated with the autophagosomes. Note that Rab7, used as a marker of the endolysosomal pathway, is involved in both the autophagic and endolysosomal pathways.

\section{Monitoring autophagic flux}

It is important to distinguish effects on lysosomal activity from effects on autophagosome formation. This problem has not previously been properly addressed in screening assays used to identify compounds modulating autophagy. The assay described by Hundeshagen and colleagues [5], by the ingenious use of tandem fluorescent tags with differential pH sensitivity, allows this discrimination. The authors use this assay successfully in a secondary screen to identify cardiac glycosides as modulators of autophagy.

Most assays for autophagy modulators use the autophagy marker protein LC3 as readout for autophagic activity. LC3 is a mammalian homolog of the yeast ATG8 protein, a ubiquitin-like protein that becomes lipidated and tightly associated with the autophagosomal membranes. LC3 is also important in the process of selective autophagy, whereby intracellular components such as protein aggregates, organelles and pathogens are removed [3]. Selective autophagy is mediated by autophagic cargo receptors such as p62, NBR1, NDP52 and NIX, which contain an LC3-interacting region (LIR) and can therefore bind directly to LC3 [3]. LC3 proteins are specifically cleaved at their carboxyl termini to form LC3-I, which has an exposed carboxy-terminal glycine that is conjugated to phosphatidylethanolamine to form LC3-II, which is tightly bound to the autophagosomal membranes and serves as an autophagic marker protein.
The most popular of the assays using LC3 are microscopy-based green fluorescent protein (GFP)-LC3 puncta formation assays and western blots of LC3-I and LC3-II forms [6]. Shvets et al. more recently pioneered the use of flow cytometry to quantify the turnover of GFP-LC3 as an assay to measure autophagic activity in living mammalian cells [7].

Hundeshagen and colleagues [5] used GFP-LC3B in an initial screen by flow cytometry to quantify autophagosome formation in response to a library of 1,120 Food and Drug Administration approved compounds. The assay uses the human breast cancer cell line MCF-7 stably expressing GFP-LC3B to quantify autolysosome formation measuring GFP fluorescence intensity in 96-well plates, and identified 38 compounds as potential activators and 36 as inhibitors of autophagy. Among the activators were several cardiac glycosides, including digoxin, strophanthidin and digoxigenin, and in the next step, these compounds were screened using a tandem fusion of the red, acid-insensitive mCherry and the acidsensitive GFP to measure formation of autolysosomes and their degradation by flow cytometry in a large cell population (Figure 2). Because of the low $\mathrm{pH}$ of the autolysosome, the green fluorescence from the acidsensitive GFP is lost on fusion of the autophagosome with the lysosome, but the red fluorescence from the acid-insensitive mCherry is not lost until the proteins are 


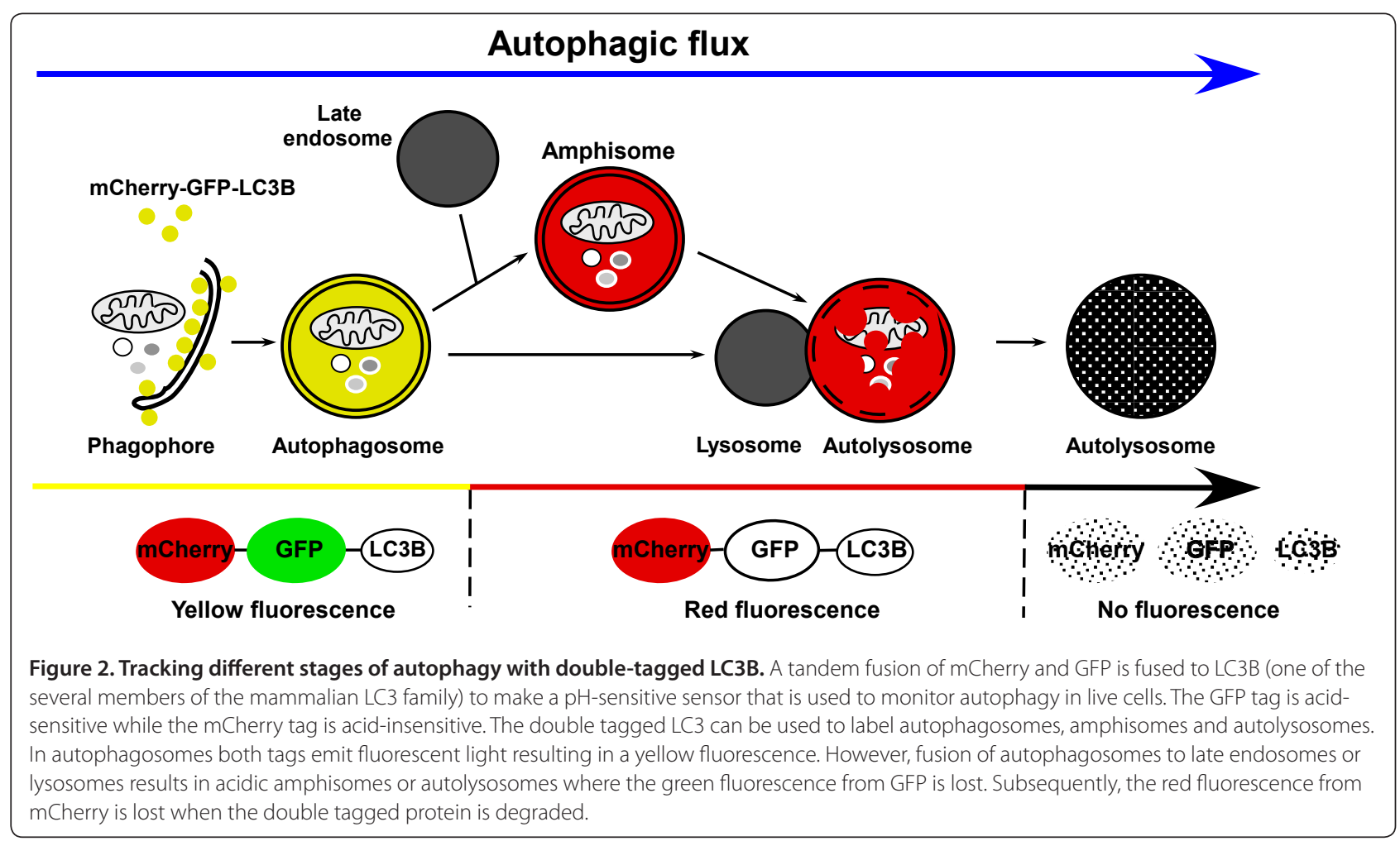

degraded in the autolysosome. The double tag strategy to distinguish autophagosomes from autolysosomes has been described before $[8,9]$, but this is the first report of its use for flow cytometry to monitor distinct steps in the autophagic pathway.

\section{The significance of cardiac glycosides, and some provisos}

A general problem when attempting to measure autophagic flux is the interconnection between the endocytic and autophagosomal pathways (see Figure 1), which makes it difficult to ensure that the observed effects are on autophagic flux and not the endolysosomal pathway. Compounds affecting late steps of the autophagic pathway may also interfere with the endocytic pathway. To address this, Hundeshagen et al. [5] used GFP-Rab7 as a marker of endolysosomal activity. Rab7 is associated with late endosomes, lysosomes and autolysosomes and is required for fusion of autophagosomes to lysosomes (see Figure 1) and is therefore not a specific indicator of endolysosomal flux. However, since the authors [5] monitor the fluorescence levels of GFP-Rab7 along with the double tagged LC3, the strategy seems reasonable and can give valuable information on effects on endolysosomal activity.

Using the double tag LC3B and the GFP-Rab7 degradation assays, Hundeshagen et al. [5] showed digoxin to be the most potent autophagy stimulator. Cardiac glycosides are used in treatment of heart failure and arrhythmia. These drugs increase the level of calcium by inhibiting the $\mathrm{Na}^{+} / \mathrm{K}^{+}$-ATPase, and thereby increase cardiac contractile force. It is known that increases in intracellular calcium induce autophagy, so it is perhaps not surprising that cardiac glycosides activate autophagy. Cardiac glycosides have been suggested for cancer therapy, and their stimulatory effect on autophagy may be important in this context.

There is one further proviso about screening strategy. Hundeshagen et al. [5] used GFP-LC3 for the initial screen and then employed mCherry-GFP-LC3 in a secondary screening, so it is not clear what the outcome would have been if the authors had used mCherry-GFPLC3 in the primary screen. The importance of the strategy chosen for the primary screen is emphasized by results from another group [10], who screened a collection of 3,584 chemicals, including the chemical library Hundeshagen et al. [5] used, and in the same cell line, without identifying the same autophagy modulators. The difference was that Balgi et al. [10] used an automated microscopy screen based on the GFP-LC3 puncta formation assay. In this screen perhexiline, niclosamide, amiodarone and rottlerin were identified as autophagy modulators [10]. Considering these different results, and the caveats and shortcomings that different assays have, it seems necessary to use a combination of different assays to perform exhaustive screens for small molecule autophagic modulators. 
That said, the work of Hundeshagen and colleagues [5] is clearly a step forward in quantitative cell population based monitoring of distinct steps of the autophagy pathway in the screening for autophagy modulators. Further development and refinement of autophagy screening protocols from this and other groups are to be expected.

\section{Acknowledgements}

This work was supported by grants from the FRIBIO program of the Norwegian Research Council, the Norwegian Cancer Society, the Aakre Foundation and the Blix Foundation to T.J.

Published: 2 June 2011

\section{References}

1. Levine B, Kroemer G: Autophagy in the pathogenesis of disease. Cell 2008, 132:27-42.

2. Mizushima N, Levine B, Cuervo AM, Klionsky DJ: Autophagy fights disease through cellular self-digestion. Nature 2008, 451:1069-1075.

3. Johansen T, LamarkT: Selective autophagy mediated by autophagic adapter proteins. Autophagy 2011, 7:279-296.
4. Mathew R, White E: Autophagy in tumorigenesis and energy metabolism: friend by day, foe by night. Curr Opin Genet Dev 2011, 21:113-119.

5. Hundeshagen P, Hamacher-Brady A, Eils R, Brady NR: Concurrent detection of autolysosome formation and lysosomal degradation by flow cytometry in a high-content screen for inducers of autophagy. BMC Biology 2011, 9:38

6. Mizushima N, Yoshimori T, Levine B: Methods in mammalian autophagy research. Cell 2010, 140:313-326.

7. Shvets E, Fass E, Elazar Z: Utilizing flow cytometry to monitor autophagy in living mammalian cells. Autophagy 2008, 4:621-628.

8. Kimura S, Noda T, Yoshimori T: Dissection of the autophagosome maturation process by a novel reporter protein, tandem fluorescenttagged LC3. Autophagy 2007, 3:452-460.

9. Pankiv $\mathrm{S}$, Clausen TH, Lamark T, Brech A, Bruun JA, Outzen H, Overvatn A Bjorkoy G, Johansen T: p62/SQSTM1 binds directly to Atg8/LC3 to facilitate degradation of ubiquitinated protein aggregates by autophagy. J Biol Chem 2007, 282:24131-24145.

10. Balgi AD, Fonseca BD, Donohue E, Tsang TC, Lajoie P, Proud CG, Nabi IR, Roberge M: Screen for chemical modulators of autophagy reveals novel therapeutic inhibitors of mTORC1 signaling. PLoS One 2009, 4:e7124

doi:10.1186/1741-7007-9-39

Cite this article as: Hansen TE, Johansen T: Following autophagy step by step. BMC Biology 2011, 9:39. 